\title{
Competencias didácticas para la formación inicial de profesores de Chile. Un análisis comparado
}

\author{
Didactic competences for the initial \\ training of teachers in Chile. A comparative analysis \\ Mónica Díaz $Z^{\mathrm{a}}$, Marcela Romero-Jeldres's, \\ Tricia Mardones ${ }^{c}$, Soledad Castillo ${ }^{D}$ \& Rosa Sequeida ${ }^{\mathrm{E}}$ \\ a Universidad Metropolitana de Ciencias de la Educación, Chile. $\triangle$ monica.diaz@umce.cl \\ [orcid.org 0000-0001-7937-326]; \\ b Universidad Metropolitana de Ciencias de la Educación, Chile. marcela.romero@umce.cl \\ [orcid.org/0000-0002-9706-9148]; \\ c Universidad Metropolitana de Ciencias de la Educación, Chile. tricia.mardones@umce.cl \\ [orcid.org/0000-0001-6706-4568]; \\ d Universidad Metropolitana de Ciencias de la Educación, Chile. soledad.castillo@umce.cl \\ [orcid.org/ 0000-0002-2540]; \\ e Universidad Católica del Norte, Chile. rosa.sequeida@ucn.cl \\ [orcid.org/0000-0003-2179-8560]
}

RESUMEN

El presente artículo es parte de una investigación mayor que responde a los desafíos del Proyecto PMI-EXA-PNNI 01-17 DIUMCE-UMCE, desarrollado en el marco del fortalecimiento de la formación de profesores que buscó mecanismos para respaldar el logro de las competencias pedagógicas inscritas en las secuencias temporales de los diseños curriculares de las carreras de pedagogía en Chile. Mediante un enfoque cualitativo interpretativo, se realiza un análisis documental para establecer las relaciones semánticas y de contenidos entre un modelo teórico chileno, que se atreve a innovar en las competencias, el Marco para la Buena Enseñanza (MBE) y los Estándares de Desempeño Docente. Los resultados dan cuenta de un correlato entre el modelo chileno y los marcos ministeriales, en lo referido a la acción profesional; no obstante, existen otros elementos de igual importancia para el fortalecimiento de la Formación Inicial Docente (FID), asociados con las competencias sociales, de comunicación y de vocación laboral que están ausentes en el Marco para la Buena Enseñanza y los Estándares de Desempeño Docente Pedagógicos, a tener en cuenta.

PALABRAS CLAVE: competencias pedagógicas, educación superior, formación inicial docente. 


\section{ABSTRACT}

This article is part of a larger investigation that responds to the challenges of the PMIEXA-PNNI 01-17 DIUMCE-UMCE Project, developed within the framework of strengthening teacher training that sought mechanisms to support the achievement of pedagogical competences inscribed in the temporal sequences of the curricular designs of the pedagogy careers in Chile. Through a qualitative interpretive approach, a documentary analysis is carried out to establish the semantic and content relationships between a Chilean theoretical model, which dares to innovate in the competences, the Framework for Good Teaching (MBE) and the Pedagogical Standards. The results show a correlation between the Chilean model and the ministerial frameworks, regarding professional action, however, there are other elements of equal importance for the strengthening of Initial Teacher Training (FID), associated with social competences, communication and vocation of work that are absent in the Framework for Good Teaching and Pedagogical Standards, important to consider.

KEY WORDS: Pedagogical Competences, Higher Education, Initial Teacher Training.

\section{INTRODUCCIÓN}

En la discusión actual a nivel nacional e internacional, es recurrente la temática sobre la calidad de la educación desde la mejora en la Formación Inicial Docente (FID). En el contexto chileno, la política de mejoramiento de la FID determina el logro de los aprendizajes esperables de los estudiantes de pedagogía al término de su proceso de formación universitaria. Al mismo tiempo, el Ministerio de Educación de Chile (Mineduc) provee de información basal para que las universidades orienten y mejoren sus procesos formativos frente a la ausencia de "un sistema articulado de regulación basado en estándares que considere la acreditación de las carreras de pedagogía, la certificación para ejercer la profesión y el registro de nuevos profesores" (Sotomayor \& Gysling, 2011, p. 117).

En Chile desde la década de los ochenta, se van instalando regulaciones que dan origen a la Evaluación Nacional Diagnóstica de la Formación Inicial Docente (END) como parte del Sistema de Desarrollo Profesional Docente, vigente en la Ley $\mathrm{N}^{\circ} 20.903$ desde el año 2016. Este marco legal establece la obligatoriedad de la aplicación de dos evaluaciones diagnósticas sobre formación inicial en las universidades que imparten pedagogía. Además de establecer mecanismos de acompañamiento y nivelación de estudiantes como requisitos para obtener la acreditación de carreras y programas. Un año antes del egreso, los estudiantes rinden la prueba como requisito de titulación, sin ser habilitante para su desempeño laboral (CPEIP, 2018).

Ahora bien, la END considera, como base de la evaluación, requerimientos disciplinares y pedagógicos, cuyo conocimiento acumulado recoge las experiencias de los Estándares de 
Desempeño para la Formación Inicial de Docentes (2001) y el Marco para la Buena Enseñanza (2008). Iniciativas que han permitido operacionalizar instrumentalmente los conocimientos mínimos del ámbito disciplinar y de la enseñanza, así como respecto de las competencias genéricas (Adaros, 2013). Ello no ha estado exento del amplio debate universitario, dado que los Estándares orientadores, de algún modo, proyectan y homogenizan lo que debe saber y qué saber hacer un docente que recién comienza a desempeñarse en el ámbito laboral (Ávalos, 2014), dando la alerta hacia la imposición de acciones evaluativas con enfoques formativostécnicos para una formación profesional docente. Pavié (2011) sostiene que asumir una homogeneización de estándares no permite, o al menos condiciona, la diversidad contextual de la formación universitaria, respecto de los modelos de formación estandarizada para la docencia instalados desde la OCDE (2010). En consecuencia, propiciar una sola perspectiva del "saber-poder hacer" resulta al menos restrictivo, entendiendo que es de suyo conocido que todo modelo tiene en su base distintas representaciones sobre qué conforma un buen docente y de qué capacidades se debe disponer para transmitir el conocimiento e impartir clases de calidad (Hallet, 2006).

Por consiguiente, para ajustarse al mandato ministerial chileno, salvaguardando los criterios específicos de evaluación para la acreditación de los programas regulares de las carreras de pedagogía (CNA, 2018), las universidades chilenas han debido transformar sus modelos educativos junto con sus diseños curriculares y programas de estudios, tomando como referencia los modelos basados en competencias, y adscribiéndose al mismo tiempo al paradigma educativo de la experiencia de Tuning en Europa, contextualizada a la realidad latinoamericana (Beneitone et al. 2007).

Esta adaptación no siempre ha ido de la mano con el espíritu planteado por la metodología Tuning, como experiencia de diálogo e integración para facilitar la comprensión de las estructuras curriculares, el reconocimiento de las titulaciones, la movilidad estudiantil y la internacionalización, cuyo paradigma educacional pone en el centro al estudiante que aprende, al tiempo que busca puntos comunes de referencias centrados en las competencias, y no en las asignaturas con el fin de respetar la diversidad, la libertad y la autonomía. Su instalación ha sido más impuesta que dialógica, por motivaciones de índole político-económica, asociada al financiamiento estatal de las universidades que les hace muchas veces perder la potestad del debate, junto con una bajada de la información que da cuenta de cierta unidireccionalidad en la relación con los cuerpos académicos (Muñoz \& Sobrero, 2006).

Ello se vislumbra por estos días en los procesos de modernización curricular, posteriores a la puesta en marcha de la metodología Tuning, y evidenciada en las demandas que imponen los procesos de acreditación, donde las resistencias, tarde o temprano, obligan a las universidades y carreras a incorporar innovaciones en sus sellos o en sus rediseños curriculares, para ajustarse a la política educacional. En ese momento, la metodología Tuning en su concepto fundamental se ha hecho acreedora de todas las adaptaciones que 
las instituciones puedan hacerle, a riesgo muchas veces de sentirla colonizadora, cuando los estándares y /o competencias se transforman en un nuevo mecanismo de control.

En tal sentido y con la idea de mantener el espíritu dialógico de Tuning, se hace preciso señalar que no da lo mismo instalar competencias genéricas y específicas consensuadas participativamente en los rediseños, en vez de hacerlas calzar instrumentalmente en los perfiles y competencias profesionales definidas según estándares nacionales e internacionales. Las competencias y los resultados del aprendizaje deben corresponderse con las cualificaciones últimas de un programa de aprendizaje, permitiendo flexibilidad y autonomía en la construcción del currículo, al mismo tiempo que sirven de base para la formulación de indicadores de nivel que puedan ser comprendidos internacionalmente (Bravo, 2005; Castillo \& Sequeida, 2019).

Por ello, buscando que los procesos mandatados por la Evaluación Nacional Diagnóstica de la Formación Inicial Docente (END), puedan permitirles a las universidades chilenas responder a los procesos de nivelación explicitados por la política educacional y, al mismo tiempo, acceder a una cierta autonomía para posibilitar la internacionalización y movilidad estudiantil. Esta investigación asociada al Proyecto PMI-EXA-PNNI 01-17 DIUMCE-UMCE, buscó establecer un modelo predictivo de competencias pedagógicas para la formación inicial docente, que permita monitorear la formación, al mismo tiempo que estudia el desarrollo de las competencias. Ello con el fin de no caer en la instrumentalización y, al mismo tiempo, tensionar la discusión pendiente sobre la didáctica en las distintas especialidades, cuando debemos desarrollar competencias específicas con carácter homogéneo para las diferentes carreras de pedagogía y su evaluación en particular.

En este artículo, se da cuenta de la primera tensión: el constructo de los modelos teóricos y/o enfoques paradigmáticos para la derivación de competencias y su relación con los dos elementos principales que tiene la política educativa chilena como el Marco para la Buena Enseñanza (MBE) y los Estándares de Desempeño Docente, en cuyas bases se encuentran las competencias genéricas y específicas para la educación planteadas desde Tuning Latinoamérica. Se espera aportar con elementos teóricos que alimenten el debate del concepto de competencias didácticas para el contexto latinoamericano y su evaluación.

\section{REFERENTES TEÓRICOS}

El uso del concepto de competencias, concebido como sustrato conceptual para el contenido del enfoque de competencias, ha tenido no solo una importancia política, sino también, de desarrollo personal, social y económico. Desde el punto de vista de la OECD (2010), los jóvenes que entran al mercado laboral necesitan competencias inmediatas para la empleabilidad, junto con un abanico de competencias cognitivas y 
laborales que les permitan afrontar contextos cambiantes en el empleo, y mantener su capacidad de aprendizaje a lo largo de su trayecto profesional. Esta información resulta de importancia para las universidades formadoras de profesionales en las distintas carreras y, más todavía, en el caso de la educación chilena, donde el mercado del trabajo está lo suficientemente regulado por la política educacional, asociada con el Sistema de Desarrollo Profesional Docente y la Evaluación Nacional Diagnóstica de la Formación Inicial Docente (END). Lo anterior impone nuevas demandas de empleabilidad al rol de las universidades para asegurar eficazmente la transición inicial al mercado del trabajo de la educación.

Lo anterior, no solo tensiona el rol de las universidades versus el mercado laboral, sino que también afecta la formación, por cuanto las competencias para la educación no están planteadas como opción de la formación profesional docente, sino para ser ejecutadas y desarrolladas con fines de control en los procesos de aseguramiento de la calidad y la acreditación de las carreras y su viabilidad financiera universitaria. Si bien estos cuestionamientos exceden los límites de este trabajo, es relevante preguntarse qué se entiende por competencias desde el punto de vista pedagógico, con el fin de tener un corpus teórico que permita orientar sin ingenuidades la reflexión del hacer, planteado en el mandato ministerial.

Méndez (2007) hace una revisión bibliográfica global del concepto y sintetiza señalando que el concepto de competencia se presenta vinculado con la noción de interrelación de diferentes elementos, articulados en función de una finalidad. Esta integración moviliza: a) habilidades que se ejercen desde los contenidos disciplinares y los saberes experienciales para resolver tareas complejas, b) recursos para la resolución de problemas y el desarrollo de competencias básicas, de perfeccionamiento o de la vida, cuya integración conduce posteriormente al desarrollo de competencias transversales y genéricas, y c) la pertinencia de los recursos puestos en juego, son medidos sobre la base del desempeño alcanzado en la resolución del problema.

Desde el punto de vista pedagógico, lo anterior se puede asociar con las competencias para reflexionar sobre la acción práctica, la reflexión docente acerca de la enseñanza, la propia acción y la conducción de la acción práctica de la enseñanza. Estos elementos son denominados por Jank y Meyer (2009) como competencias didácticas. Según los autores, ellas se desarrollan gradualmente a través del estudio teórico y de actividades prácticas en ámbitos pedagógicos por medio de la socialización profesional. Son integrales en la medida que fusionan las tres dimensiones de la praxis: pensar-sentir-actuar. Son transmitidas biográficamente como un flujo directo de la estructura de personalidad del profesor y su trayectoria profesional previa, pueden ser diferenciadas y mejoradas a través de la ejercitación, y ser debilitadas a través de los cambios en las condiciones laborales, problemas de salud, aplicación de rutinas nefastas, entre otros. En tal sentido, no sería posible actuar didácticamente sin reflexionar didácticamente. 
A partir de estos planteamientos teóricos, Košinár (2009) señala que es posible encontrar en la literatura alemana tres enfoques teóricos profesionales diferentes: a) el enfoque teórico-estructural que define los requisitos específicos y teóricos de la estructura, el problema de la acción profesional y los requisitos en la profesión docente. Manteniendo como tensión del enfoque la suposición de la acción del maestro entre la incertidumbre y la previsibilidad; b) el enfoque teórico de la competencia que se basa en la organización de los procesos sucesivos del aprendizaje escolar. Quienes se adscriben a este enfoque se preguntan respecto de qué competencias (conocimiento y habilidad) y disposiciones (contratación), deben traer los profesores y ser capaces de manejar al realizar las tareas profesionales; y c) el enfoque biográfico ocupacional busca que la profesionalidad sea probada en el proceso, es decir, el modo para formarse y convertirse en profesor se realiza sobre la base de la experiencia biográfica pasada (profesional), siempre conectada con la personalidad, la autoimagen profesional y la disposición para lidiar con desafíos.

Ahora bien, sobre la base de estos enfoques, es posible revisar algunos modelos heurísticos de competencia profesional docente que orientan la formación. Uno de ellos se refiere al modelo de competencia profesional del profesorado (resultado del programa de investigación COACTIV) de Kunter y Baumert (2006) y Kunter et al. (2008) que se centra en el conocimiento profesional en los campos de especialización, conocimiento didáctico y conocimiento pedagógico-psicológico, buscando un diseño "permanentemente exitoso" del sistema cognitivo, con lecciones activadoras y motivadoras para los estudiantes. Se le critica que las áreas de competencia y facetas están desconectadas unas de las otras.

En segundo lugar, el modelo de habilidades educativas, similar al anterior, que se apoya en una "taxonomía de competencias educativas" para las habilidades didácticas y específicas y agrega dos áreas más de habilidades pedagógicas básicas y habilidades pedagógicas especializadas importantes para el ejercicio de la profesión docente. Respecto de las competencias básicas, Bauer et al. (1996) y Bauer (2005) señalan que forman parte del repertorio de acción del profesorado e incluyen: habilidades de diagnóstico, habilidades mentales, planificación y habilidades de interacción, planteando la necesidad de una mayor educación en el área de la comunicación, la cooperación y el trabajo en equipo en los grupos docentes.

En tercer lugar, el modelo de competencia educativa de Nieke (2012), que se nutre del concepto de antropología pedagógica, planteada por Heinrich Roth, quien ya en 1976 propone la competencia técnica, la competencia social y autocompetencia, como elementos requeridos para la ciencia educativa, en cuyo sentido instalan como relevantes, las condiciones histórico-sociales. En base a ello, Nieke (2012) desarrolla un esquema estructural de la competencia de acción pedagógica que plantea un contexto general sistemático para definir cuatro componentes: el primer componente se refiere al Análisis de la sociedad, para dar cuenta que las tareas educativas surgen en un contexto social, por 
lo que no deben resolverse de manera aislada en las instituciones educativas, sino que siempre insertas en un contexto social y político. El segundo componente, Diagnóstico de la situación también traducido como diagnóstico de la sociedad, se refiere a las acciones específicas en el espacio social, posibilitando las condiciones para abordar las necesidades de los estudiantes. Este componente cuenta con cuatro dimensiones: (a) la corporalidad involucrada en la acción educativa, (b) el aparato mental (identidad y el autoconcepto de los estudiantes), (c) el espacio (influencia del medio ambiente y el espacio en la acción educativa), y (d) la interacción (secuencia constante de acciones y contra-acciones con significado simbólico entre profesores y estudiantes). El tercer componente Autorreflexión incluye cuestiones éticas, autoimágenes, objetivos profesionales e intenciones, y se articula en dos dimensiones: (a) el autoconcepto profesional, que implica la búsqueda de los principios rectores (¿Quién soy yo como maestro? ¿Qué quiero lograr en mi carrera profesional? ¿Qué máximas orientan mi actuar profesional? ¿Cómo puedo medir el efecto de mis acciones? etc.) y (b) preocupación/afectación por/de sí mismo, porque el profesor está inevitable y directamente involucrado en la acción profesional. Finalmente, un cuarto componente, asociado a la acción pedagógica denominada Acción profesional, cuyos fundamentos son intersubjetivos y metodológicamente comprobables. Las teorías de la acción profesional en su mayoría incluyen las siguientes cinco fases: (a) determinación del objetivo, (b) el diagnóstico de la situación de la acción, (c) establecer un plan de acción, a través del repaso virtual de varias alternativas, decidiendo justificadamente por una de ellas, (d) la acción, la realización de la acción mediante la actualización de los patrones ya ensayados y (e) la evaluación y comprobación de las acciones exitosas.

En este sentido, los autores Romero-Jeldres y Faouzi (2018) definen un constructo de competencias didácticas, a partir de los aportes de los modelos y enfoques alemanes, específicamente el de Nieke (2012), pero adicionan dos componentes nuevos para construir un núcleo teórico que se adecue a la realidad chilena: un primer componente que se relaciona con las competencias sociales y comunicativas, asumidas desde los aportes de Erpenbeck y Von Rosenstiel (2007) y de Hortsch y Kersten (2010), con el fin de establecer las capacidades necesarias para mantener relaciones adecuadas con otros y conformes a las circunstancias, sumando la comunicación, la cooperación, la capacidad de enfrentarse a los conflictos y la capacidad de comprender las competencias y los intereses de los demás. El segundo componente que adicionan los autores está referido a la vocación laboral, aportado desde Mórtola (2011), Dubet (2010) y Alliaud (1993), cuyas narrativas permiten relevar tres focos vocacionales, pertenecientes a la cultura pedagógica y a las formas en que viven el trabajo los docentes de Latinoamérica (Fig. 1).

En la Fig. 1 se puede ver el constructo Modelo teórico Competencia Didáctica Profesional, validado por Romero-Jeldres y Faouzi (2018, 2020), con docentes chilenos sin formación pedagógica provenientes de liceos o instituciones de Educación Media TécnicoProfesional, que se desempeñan en contextos de alta vulnerabilidad. Este marco se nutre 
Fig. 1. Modelo teórico de competencia didáctica profesional.

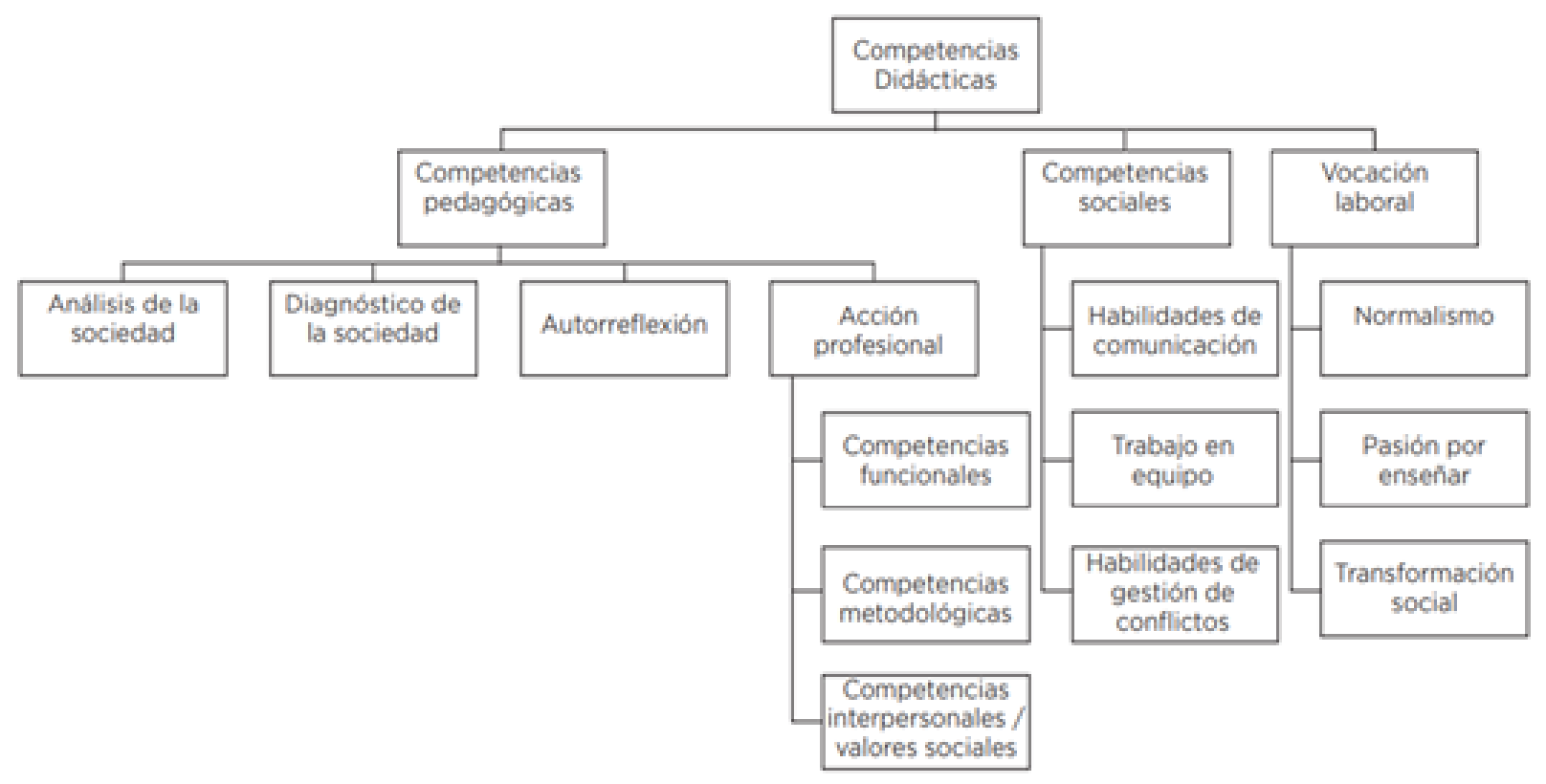

Fuente: Romero-Jeldres y Faouzi (2018, 2020).

de un enfoque teórico estructural, un modelo sistémico con base en la antropología pedagógica, más los aportes histórico-sociales y las narrativas vocacionales para el contexto latinoamericano. Cabe preguntarse, si ese mismo modelo teórico tiene validez predictiva cuando se utiliza con profesores en formación. En esta investigación, se da cuenta del correlato que tiene este constructo cuando se le compara con los lineamientos emanados por la política educativa, a saber, el Marco para la Buena Enseñanza (MBE) y los Estándares Orientadores para la Formación Inicial Docente.

\section{METODOLOGÍA}

Este estudio se emprende desde un método científico cualitativo, cuyo enfoque interpretativo sigue los lineamientos planteados por Vasilachis de Gialdino (2006), buscando ubicar al científico en contextos reales, para acceder a las estructuras de significado. El diseño del estudio es documental de corte transversal, caracterizado por la utilización de fuentes documentales que no sean de observación o de conversación (Valles, 2009). El corpus analizado fue el Marco para la Buena Enseñanza (2008), y los Estándares Orientadores para Carreras de Educación Parvularia (2012), Estándares Orientadores para Egresados de Carreras de Pedagogía en Educación Básica (2012), Estándares Orientadores para Egresados de Carreras de Pedagogía en Educación Media (2012), Estándares para la Formación Inicial de los Profesores de Educación Física (2014), Estándares para la Formación Inicial de los Profesores de Educación Especial (2014), 
Estándares para la Formación Inicial de los Profesores de Inglés (2014) y los Estándares Orientadores para Egresados de Carreras de Pedagogía en Artes Visuales y Música (2014).

Para el análisis documental, se utilizó el análisis de contenido, considerando el objetivo descriptivo que busca establecer las relaciones semánticas y de contenidos entre un modelo teórico chileno que se atreve a innovar en las competencias pedagógicas para la formación de profesores, el Marco para la Buena Enseñanza y los Estándares de Desempeño Docente Pedagógicos mandatados por el Mineduc. Según Piñuel (2002), el nombre de análisis de "contenido" supone que el "contenido" se encuentra oculto dentro de un "continente" (los textos donde se encuentra la información), por lo cual al ser examinado "por dentro" permite desvelar su contenido, es decir, su significado o sentido. En esta investigación, se recurrió a la vertiente cualitativa con categorías apriorísticas, las cuales son erigidas antes de la compilación de la información, es decir, las temáticas surgen desde los objetivos de investigación, mediante la indagación teórica que sustenta el estudio (Cisterna, 2005). En los enunciados correspondientes a las unidades de análisis, se estableció la presencia o ausencia de una cualidad del contenido (Porta \& Silva, 2003; Díaz, 2018), basándose en el análisis interno de los documentos, pretendiendo enfatizar el sentido del enunciado (López, 2002). Para la realización de este procedimiento metodológico, se consideraron tres fases de análisis presentadas a continuación.

En la primera fase, se construyó una matriz de análisis que consideró las dimensiones teóricas del constructo Competencia Didáctica profesional, validado por los autores (Romero-Jeldres \& Faouzi, 2018, 2020) como categorías apriorísticas, donde las competencias didácticas las componen dos dimensiones: Competencias Pedagógicas y Competencias Sociales. La primera dimensión está formada por cuatro subcategorías como análisis de la sociedad, diagnóstico de la sociedad (también traducido como diagnóstico de la situación), autorreflexión y acción profesional. Esta última subcategoría compuesta por competencias funcionales, competencias metodológicas y competencias interpersonales/ valores sociales. La segunda dimensión está compuesta por habilidad de comunicación, trabajo en equipo y habilidad de gestión de conflictos.

En la segunda fase, se seleccionaron las unidades de análisis que estimaron los dominios del Marco para la Buena Enseñanza tales como: Dimensión A "preparación para la enseñanza", Dominio B "creación de un ambiente propicio para el aprendizaje", Dominio C "enseñanza para el aprendizaje de todos los estudiantes" y el Dominio D "responsabilidades profesionales". Luego, las unidades de análisis correspondientes a los Estándares Orientadores para la Formación Inicial Docente, comunes a las Carreras de Educación Básica, Educación Media y Educación Parvularia, consideraron los Estándares de Desempeño Docente Pedagógicos del número 1 al 10.

La tercera fase consideró la aplicación del análisis de contenido cualitativo, identificando la presencia o ausencia de las categorías apriorísticas en las unidades de análisis estudiadas, atendiendo a los rasgos semánticos presentes en los enunciados (Fig. 2). 
Fig. 2. Desarrollo Análisis de Contenido Cualitativo Apriorístico.

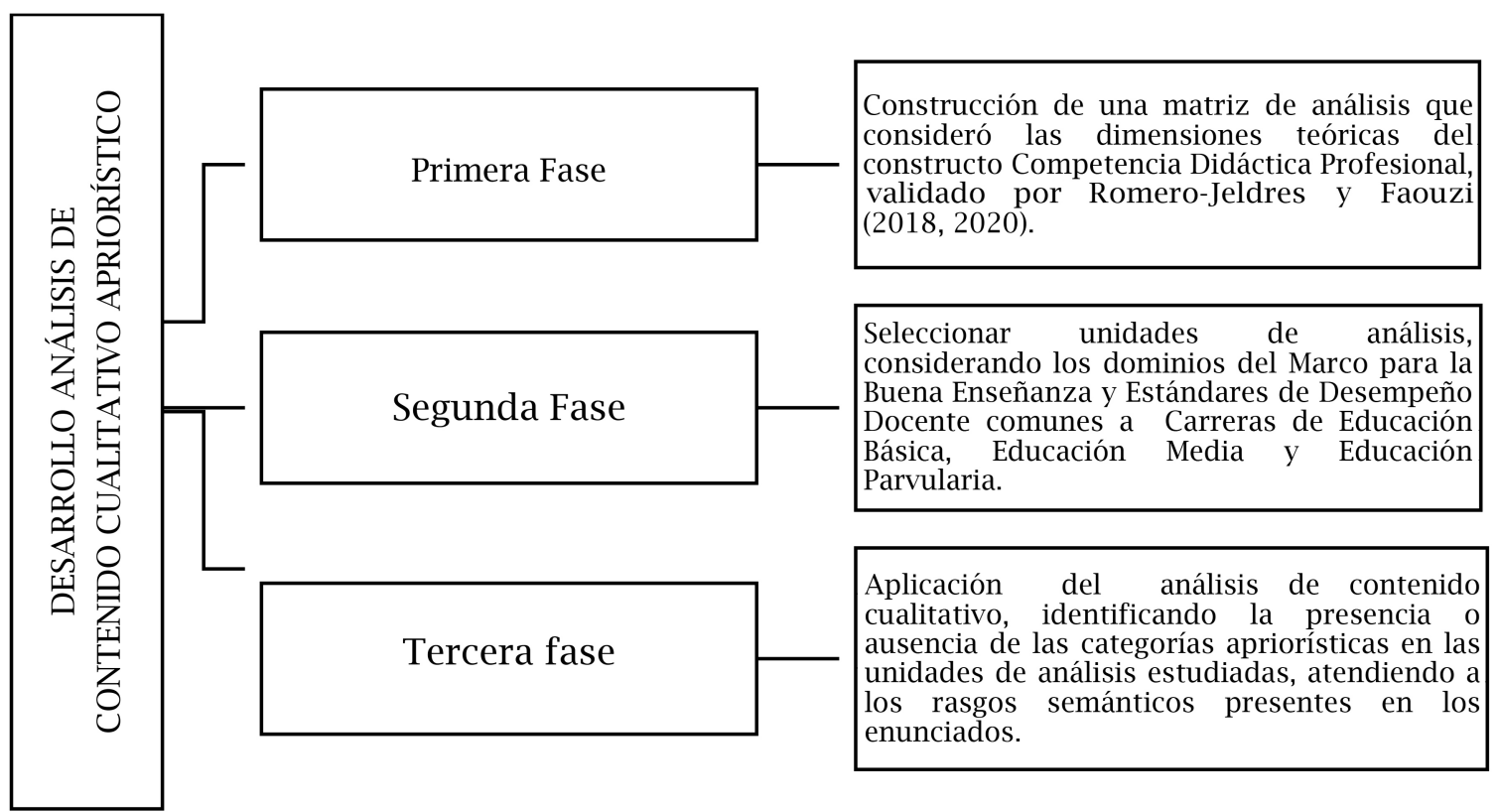

Fuente: Elaboración propia.

\section{RESULTADOS}

Los resultados observados de los análisis propuestos anteriormente exponen, en primer lugar, los aspectos asociados al Marco para la Buena Enseñanza (MBE) y describen la presencia o ausencia del constructo Competencia Didáctica Profesional, validado por RomeroJeldres y Faouzi $(2018,2020)$ en el MBE, identificando los rasgos semánticos presentes en cada dominio de estos referentes curriculares. Posteriormente, este mismo procedimiento analítico se muestra en los enunciados correspondientes a los Estándares Orientadores para la Formación Inicial Docente.

\section{MARCO PARA LA BUENA ENSEÑANZA}

Respecto del Dominio A: Preparación para la enseñanza, referido tanto a la disciplina que enseña el profesor o profesora, como a los principios y competencias pedagógicas necesarios para organizar procesos de enseñanza. Se pudo observar que la presencia del Modelo de Competencias Didácticas para Profesionales de EMTP prevalece más en lo referido a las competencias pedagógicas para la Acción Profesional, las cuales se encuentran en los criterios A1, A3, A4 y A5 (Fig. 3). Dentro de esta categoría los criterios seleccionados se asocian a las competencias metodológicas. Es importante reparar que la competencia social no se encuentra presente en ninguno de los criterios pertenecientes a este Dominio. 
Respecto del Dominio B: Creación de un ambiente propicio para el aprendizaje, este dominio hace referencia al entorno del aprendizaje en su sentido más amplio, es decir, al ambiente y clima que genera el docente, en el cual tienen lugar los procesos de enseñanza y aprendizaje. Al igual que en el dominio anterior, la mayor prevalencia se da en la competencia pedagógica para la Acción Profesional, encontrándose en los criterios B2, B3 y B4 (Fig. 3). Dentro de esta categoría los criterios seleccionados se asocian a las competencias funcionales.

En relación al Dominio C: Enseñanza para el aprendizaje de todos los estudiantes, se observa que este dominio pone en juego todos los aspectos involucrados en el proceso de enseñanza que posibilitan el compromiso real de los alumnos/as con sus aprendizajes. En la totalidad de este dominio, se encuentra presente la competencia pedagógica para la Acción Profesional. Los criterios se pueden diferenciar en: Competencias metodológicas los criterios C2 y C3; Competencias funcionales los criterios C1, C4, C5, C6 (Fig. 3).

Fig. 3. Criterios del MBE asociados a la Competencia Pedagógica "Acción Profesional".

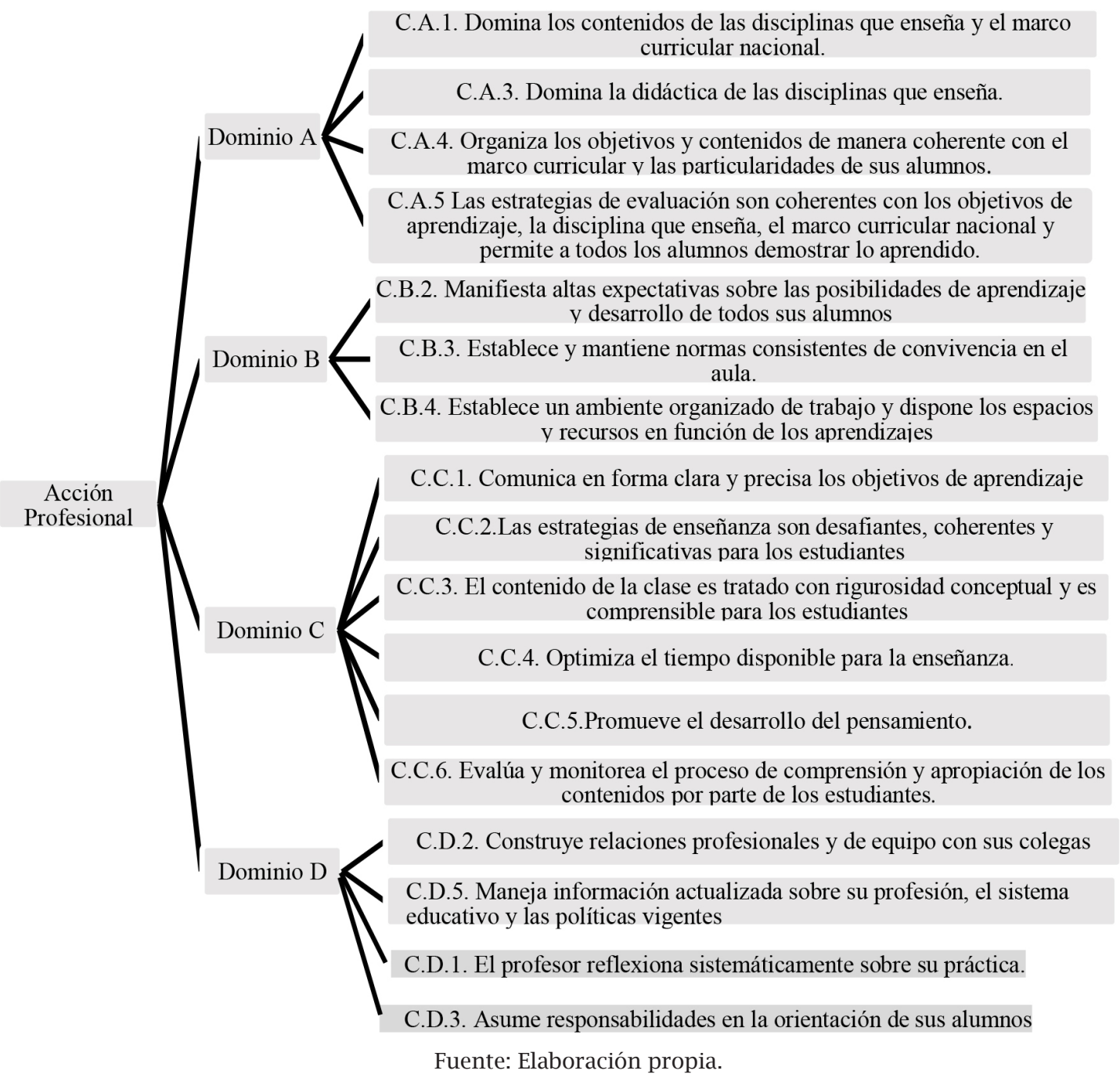


Finalmente, sobre el Dominio D: Responsabilidades profesionales, se puede mencionar que los elementos que componen este dominio están asociados a las responsabilidades profesionales del profesor. El principal propósito y compromiso de las responsabilidades profesionales sería la contribución a que todos los estudiantes aprendan. En este dominio, se observa en mayor presencia las competencias pedagógicas para la Acción Profesional, presentándose en los criterios D1, D2 y D5 (Fig. 3). Sin embargo, a diferencia de los criterios anteriores, estos se asocian con las competencias interpersonales. Finalmente, la Competencia Social se encuentra solo en el criterio D3 (Fig. 3).

\section{ESTÁNDARES PEDAGÓGICOS}

Al hacer el análisis respecto de la presencia del constructo Competencia Didáctica Profesional, validado por Romero-Jeldres y Faouzi $(2018,2020)$ en los Estándares de Desempeño Docente Pedagógicos, se pudo visualizar que en el componente Competencias Pedagógicas la subdimensión Acción Profesional, se encuentra con mayor prevalencia, presente en el estándar 1, 3, 4, 5, 6, 7 y 9 (Fig. 4). Dentro de la Acción Profesional, los estándares E3, E4 y E6 están asociados a las competencias metodológicas, los estándares E1, E5 y E7 están asociados a las competencias funcionales y, por último, el E9 se encuentra asociado a las competencias interpersonales. Es relevante señalar que al igual que en el análisis anterior, las Competencias Sociales del constructo, se encuentran ausentes en los Estándares.

Fig. 4. Estándares Pedagógicos asociados a la Competencia Pedagógica “Acción Profesional”.

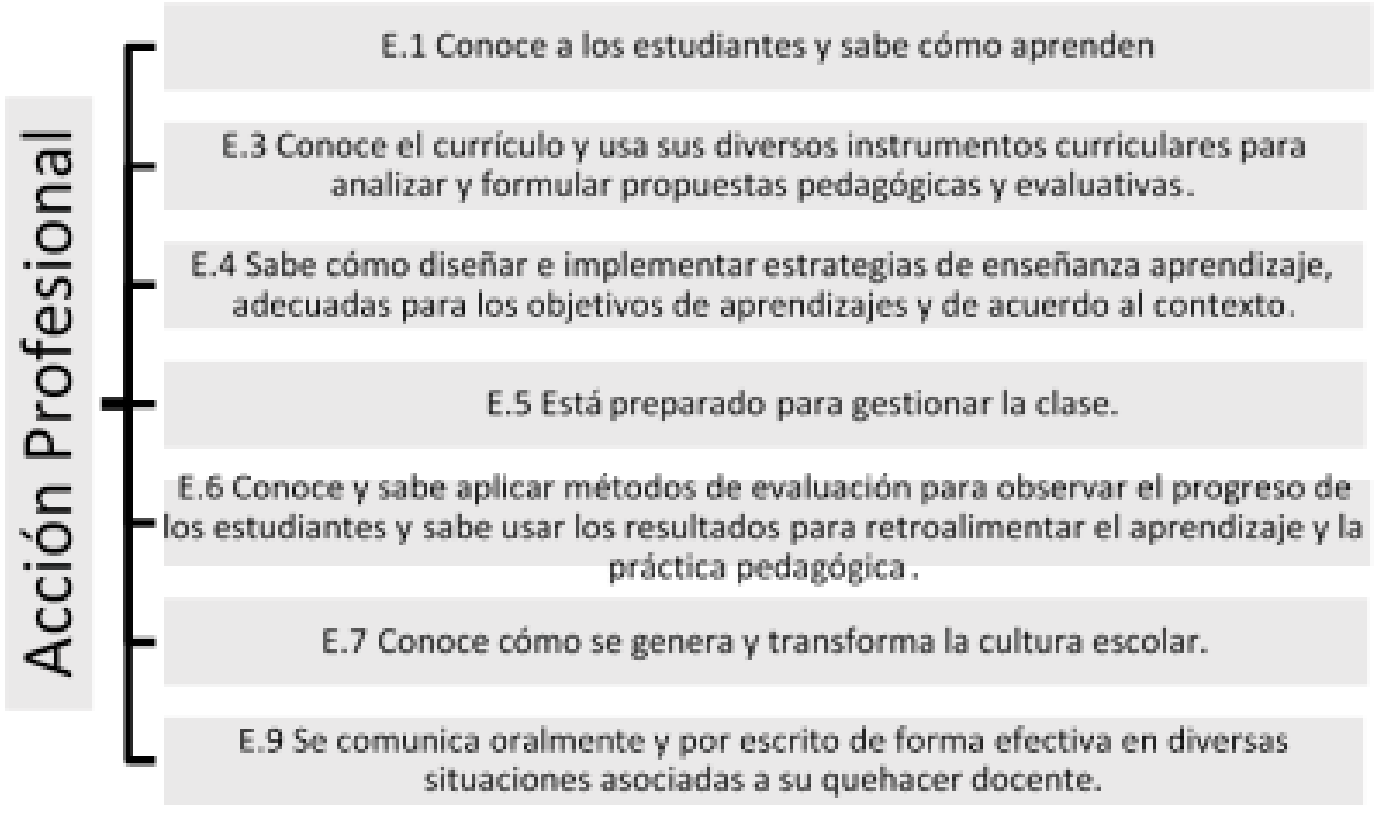

Fuente: Elaboración propia. 


\section{CONCLUSIONES}

Estos resultados son el primer insumo de una investigación mayor que busca desarrollar un modelo de competencias didácticas para el fortalecimiento de la FID, que propenda a la autonomía de las universidades para la construcción del conocimiento en la Formación Inicial Docente. Los hallazgos parciales de la investigación, por medio del análisis de contenido, dan cuenta de la presencia o ausencia de las competencias del modelo de Competencias Didácticas para Profesionales de EMTP de los autores RomeroJeldres y Faouzi (2018, 2020), en el corpus de los distintos dominios del Marco para la Buena Enseñanza (MBE) y los Estándares de Desempeño Docente Pedagógicos. Los análisis permitieron observar que las competencias pedagógicas del modelo chileno en cuestión subyacen en los distintos criterios del MBE y los Estándares de Desempeño Docente Pedagógicos, siendo la Acción Profesional la competencia más observada y las ausentes dicen relación con la Vocación laboral, la Competencia Social y Comunicativa.

Respecto de una mayor prevalencia de las competencias asociadas a la Acción Profesional en los Estándares de Desempeño Docente Pedagógicos, puede ser comprendida a partir de la formulación que el Mineduc hace de ellos, declarando que las competencias asociadas a éstos son "conocimiento del currículo, diseño de procesos de aprendizaje y evaluación para el aprendizaje. Se incluye en ellos, la dimensión moral de su profesión: que los futuros profesores y profesoras estén comprometidos con su profesión, con su propio aprendizaje y con el aprendizaje y formación de sus estudiantes" (MINEDUC, $\left.2014^{\mathrm{a}}, 2014^{\mathrm{b}}, 2014^{\mathrm{c}}, 2014^{\mathrm{d}}, 2012^{\mathrm{a}}, 2012^{\mathrm{b}}, 2012^{\mathrm{c}}\right)$.

Respecto de las Competencias Específicas Tuning en Educación se puede señalar que el modelo hace referencia a aquellas habilidades requeridas para el ejercicio profesional y disciplinar, y de aplicación en el aula o la institución; las menos importantes se refieren a aquellas de proyección social o comunitaria y en relación con aspectos históricos y culturales. Se da más importancia a los aspectos profesionalizantes en relación con la disciplina y con los aspectos didácticos, que a las competencias vinculadas con la proyección social y cultural de la profesión docente (Beneitone et al. 2007). Estos resultados son coincidentes con los obtenidos en el análisis de las competencias del modelo en los distintos dominios del MBE y Estándares de Desempeño Docente Pedagógicos, donde se da cuenta de la ausencia de las competencias sociales y la vocación laboral. Sin embargo, una definición más amplia de competencia las incorpora señalándolas como "un conjunto de conocimientos, procedimientos y actitudes combinados, coordinados e integrados para «saber hacer»y «saber estar» en el ejercicio profesional, el dominio de estos saberes permite ser «capaz de» actuar con eficacia en situaciones profesionales cambiantes" (Perales et al. 2014, p. 10).

En función a lo escrito, el término competencia tendría que ser entendido como un constructo “complejo e integrado de saberes" (Perales et al. 2014), sobre el que se 
reflexiona y, a partir del cual, se puede desarrollar un modelo de aprendizaje, destacando su carácter dinámico, holístico y situacional, y así sistematizar a nivel conceptual, sus aportes como resultado de procesos de reflexión dinámicos y enriquecedores. En esta lógica, las competencias "implican el desarrollo de capacidades que constituyen conjuntos de saberes integrados a las competencias" (Trujillo, 2014, p. 314), donde las competencias profesionales no pueden ser aisladas, sino asociadas al desempeño profesional. En este sentido cobra valor hablar de la Competencia Didáctica Profesional planteada por RomeroJeldres y Faouzi $(2018,2020)$.

Por tanto, es posible señalar que existe un correlato entre el modelo chileno y los diferentes elementos establecidos (Marco para la Buena Enseñanza y Estándares de Desempeño Docente, disciplinares y pedagógicos para las carreras de pedagogía); no obstante, el modelo planteado por Romero-Jeldres y Faouzi $(2018,2020)$ no se queda solo en la acción profesional, sino que además aborda el componente social y comunicativo, incorporando lo histórico-social y, por tanto, generando condiciones para el trabajo en las realidades latinoamericanas. Cabe preguntarse si estos dos componentes del modelo recogen la realidad del aula que viven los profesores de educación media técnica profesional y si ello es traspasable teóricamente, como insumo de contexto, a la Formación Inicial Docente cuando inician su proceso de formación.

Ahora bien, dado que la competencia vocación laboral es la gran ausente en este análisis, queda pendiente investigar si ello es relevante para la empleabilidad o basta con tener competencias funcionales a la docencia. Lo anteriormente señalado y evidenciado en este artículo, permite hacer notar que los Estándares de Desempeño Docente, disciplinares y pedagógicos y el Marco para la Buena Enseñanza solo consideran aspectos relacionados con la acción profesional, dejando ausentes las competencias sociales y la vocación laboral y ello, al instrumentalizarse, bordea lo técnico, dejando lo profesional de la toma de decisiones de lado.

Es importante señalar que el Marco para la Buena Enseñanza es la base de la evaluación docente, por lo que puede considerarse igualmente la base para los Estándares Pedagógicos. Por otro lado, los estándares disciplinarios y pedagógicos pueden considerarse como una definición más específica de los conocimientos y habilidades que deberían desarrollarse durante el proceso de formación. Es por esta razón que estas herramientas debieran considerar competencias asociadas al ámbito social y comunicacional que permitan una formación inicial más integral, dando mayor consistencia al proceso de desarrollo profesional de los docentes.

Del mismo modo, el análisis de los Estándares de Desempeño Docente, disciplinares y pedagógicos con el Modelo permite reconocer nuevas necesidades formativas que podrían significar importantes avances y mejoras en la FID con el fin de incorporar experiencias de aprendizaje, orientadas al desarrollo de las competencias sociales y comunicativas, donde el modelo chileno validado cobra valor, al mismo tiempo que se abre el debate para 
poner en discusión el concepto de Competencia Didáctica Profesional, hasta ahora poco abordado como el eje formativo para el desempeño profesional de la labor docente en cualquier ámbito donde se deba impartir formación.

\section{AGRADECIMIENTOS}

Los resultados de esta publicación han sido posibles gracias al financiamiento otorgado por DIUMCE de la Universidad Metropolitana de Ciencias de la Educación, a través del Proyecto PMI-EXA-PNNI 01-17, dirigido por la Dra. Marcela Romero Jeldres.

\section{REFERENCIAS}

Adaros, M. (2013). Implicancias de los Nuevos Estándares Pedagógicos para el Desarrollo de la Competencia Emocional en la Formación Inicial del Profesorado. Temas de Educación, 19(1), 7-24.

Alliaud, A. (1993). El maestro como categoría social: génesis y desarrollo en Argentina (1880-1915). Buenos Aires: CEAL.

Ávalos, B. (2014). La formación inicial docente en Chile: Tensiones entre políticas de apoyo y control. Estudios Pedagógicos, 40, 11-28. https://doi:10.4067/S0718-07052014000200002

Bauer, K. O. (2005). Pädagogische Basiskompetenzen. Juventa-Verl.

Bauer, K., Kopka, A., \& Brindt, S. (1996). Pädagogische Professionalität und Lehrerarbeit. Eine qualitative empirische Studie über professionelles. Handeln und Bewusstsein. Weinheim und München: Juventa.

Beneitone, P., Esquetini, C., González, J., Maletá, M., Siufi, G., \& Wagenaar, R., Proyecto Tuning América Latina; Rijksuniversiteit Groningen; Universidad de Deusto (2007). Reflexiones y perspectivas de la Educación Superior en América Latina: informe final Proyecto Tuning América Latina: 2004-2007. Bilbao: Universidad de Deusto; Groningen: Universidad de Groningen. Disponible en: http://tuningacademy.org/wp-content/uploads/2014/02/ TuningLAIII_Final-Report_SP.pdf

Bravo, N. (2005). Competencias Proyecto Tuning-Europa Tuning en América Latina. 2004. Madrid: ANECA.

Castillo, P., \& Sequeida, R. (2019). Competencias Pedagógicas en la Formación Inicial Docente. Revista Electrónica de Investigación en Docencia Universitaria 1(1),1-15.

Cisterna, F. (2005). Categorización y triangulación como procesos de validación del conocimiento en investigación cualitativa. Theoria, 14(1), 61-71.

Comisión Nacional de Acreditación (2018). Carreras de pedagogía: análisis de fortalezas y debilidades en el escenario actual. Serie Estudios sobre Acreditación. Disponible en la red: http://www.investigacion.cnachile.cl/

CPEIP (2018). Resultados Evaluación Nacional Diagnóstica de la Formación Inicial 
Docente 2017. Ministerio de Educación. Disponible en: https://www.cpeip.cl/wp-content/ uploads/2018/07/Informe-nacional-Evaluaci\%C3\%B3n-Nacional-Diagn\%C3\%B3stica-de-laFID.pdf

Díaz, C. (2018). Investigación cualitativa y análisis de contenido temático. Orientación intelectual de revista Universum. Revista General de Información y Documentación, 28(1), 119-142. https://doi.org/10.5209/RGID.60813

Dubet, F. (2010). Crisis de la transmisión y declive de la institución. Revista Política y Sociedad, 47(2), 15-25.

Erpenbeck, J., \& Von Rosenstiel, L. (2007). Handbuch Kompetenzmessung. 2. Vorbemerkung zur 2. Auflage. Stuttgart: Schäffer-Poeschel.

Hallet, W. (2006). Didaktische Kompetenzen. Lehr -und Lernprozesse erfolgreich gestalten. Stuttgart: Klett Lernen und Wissen GmbH.

Heinrich, R. (1976). Pädagogische Anthropologie - Mechanismus einer Praxis.Bd. 1: Bildsamkeit und Bestimmung. Editorial: Hermann Schroedel Verlag.

Hortsch, H., \& Kersten, S. (2010). Didaktik der Berufsbildung, Merkblätter. Dresde: SFPSWissenschaftlicher Fachverlag.

Jank, W., \& Meyer, H. (2009). Didaktische Modelle. Berlín: Cornelsen Verlag.

Košinár, J. (2009): Körperkompetenzen und Interaktion inpädagogischen Berufen. Konzepte - Training - Praxis. Bad Heilbrunn: Klinkhardt.

Kunter, M., \& Baumert, J. (2006). Who is the expert? Construct and criteria validity of student and teacher ratings of instruction. Learning Environ Res 9, 231-251. https://doi. org/10.1007/s10984-006-9015-7

Kunter, M., Tsai, Y.-M., Klusmann, U., Brunner, M., Krauss, S., \& Baumert, J. (2008). Students' and mathematics teachers' perceptions of teacher enthusiasm and instruction. Learning and Instruction, 18(5), 468-482. https://doi.org/10.1016/j.learninstruc.2008.06.008

López, F. (2002). El análisis de contenido como método de investigación. Revista de Educación, 21(4), 167-179.

Méndez, A. (2007). Terminología pedagógica específica al enfoque por competencia: el concepto de competencia. Revista Innovación educativa, 17, 173-184.

Ministerio de Educación (2008). Marco para la Buena Enseñanza. Santiago de Chile. Disponible en: https://www.docentemas.cl/docs/MBE2008.pdf

Ministerio de Educación (2012a). Estándares Orientadores para Carreras de Educación Parvularia. Santiago de Chile. Disponible en: https://bibliotecadigital.mineduc.cl/discover? scope $=\% 2$ F\&query=Est\%C3\%A1ndares+Orientadores+para+Egresados + de + Carreras + de + Peda gog\%C3\%ADa

Ministerio de Educación (2012b). Estándares Orientadores para Egresados de Carreras de Pedagogía en Educación Básica. Santiago de Chile. Disponible en: https://bibliotecadigital. mineduc.cl/discover?scope=\%2F\&query=Est\%C3\%A1 ndares+Orientadores+para+Egresados + de+Carreras+de+Pedagog\%C3\%ADa 
Ministerio de Educación (2012c). Estándares Orientadores para Egresados de Carreras de Pedagogía en Educación Media. Santiago de Chile. Disponible en: https://bibliotecadigital. mineduc.cl/discover?scope=\%2F\&query=Est\%C3\%A1ndares+Orientadores+para+Egresados + $\mathrm{de}+$ Carreras + de+Pedagog\%C3\%ADa

Ministerio de Educación (2014a). Estándares Orientadores para Egresados de Carreras de Pedagogía en Artes Visuales y Música. Santiago de Chile. Disponible en: https:// bibliotecadigital.mineduc.cl/discover?scope $=\% 2 \mathrm{~F} \&$ query $=$ Est\%C3\%A1ndares+Orientadores $+\mathrm{p}$ ara+Egresados+de+Carreras+de+Pedagog\%C3\%ADa

Ministerio de Educación (2014b). Estándares para la Formación Inicial de los Profesores de Educación Física. Santiago de Chile. Disponible en: https://bibliotecadigital.mineduc.cl/di scover? scope $=\% 2 \mathrm{~F} \&$ query $=$ Est $\%$ C3\%A1ndares+Orientadores+para+Egresados $+\mathrm{de}+$ Carreras $+\mathrm{d}$ e+Pedagog\%C3\%ADa

Ministerio de Educación (2014c). Estándares para la Formación Inicial de los Profesores de Educación Especial. Santiago de Chile. Disponible en: https://bibliotecadigital.mineduc.cl/ discover?scope=\%2F\&query=Est\%C3\%A1ndares+Orientadores+para+Egresados+de+Carreras + de+Pedagog\%C3\%ADa

Ministerio de Educación (2014d). Estándares para la Formación Inicial de los Profesores de Inglés. Santiago de Chile. Disponible en: https://bibliotecadigital.mineduc.cl/discover?sc ope $=\% 2 \mathrm{~F} \&$ query $=$ Est $\%$ C3\%A1ndares+Orientadores+para+Egresados + de + Carreras + de + Pedago g\%C3\%ADa

Mórtola, G. (2011). Breve ensayo sobre la vocación de los maestros y maestras en Argentina. Buenos Aires: Centro Editor de América Latina.

Muñoz, A.L., \& Sobrero, V. (2006). Proyecto Tuning en Chile: análisis de procesos de internacionalización de la educación superior. Revista Calidad en la Educación, 24, 249-271. http://dx.doi.org/10.31619/caledu.n24.278

Nieke, W. (2012). Kompetenz und Kultur. Beiträge zur. Orientierung in der Moderne. Wiesbaden: Springer.

OECD (2010). Synthesis Report of the OECD Reviews of Vocational Education and Training, Learning for Jobs. Series: OECD Reviews of Vocational Education and Training.

Pavié, A. (2011). Formación docente: hacia una definición del concepto de competencia profesional docente. Revista Electrónica Interuniversitaria de Formación del Profesorado, 14, 67-80.

Perales, F., Cabo, J., Vilchez, J., Fernández, M., González, F., \& Jiménez, P. (2014). La reforma de la formación inicial del profesorado de ciencias de secundaria: propuesta de un diseño del currículo basado en competencias. Enseñanza de las Ciencias, 32(1), 9-28. https:// doi.org/10.5565/rev/ensciencias.898

Piñuel, J. (2002). Epistemología, metodología y técnicas del análisis de contenido. Estudios de Sociolingüística, 3(1), 1-42.

Porta, L., \& Silva, M. (2003). La investigación cualitativa: El Análisis de Contenido en la 
investigación educativa. Anuario Digital de Investigación Educativa, 14, 1-18.

Romero-Jeldres, M., \& Faouzi, T. (2018). Validación de un modelo de competencias pedagógicas para docentes de Educación Media Técnica. Educación y Educadores, 21(1), 114132. https://dx.doi.org/10.5294/edu.2018.21.1.6

Romero-Jeldres, M. R., \& Faouzi-Nadim, T. (2020). Modelo estructural de competencia profesional didáctica para profesores técnicos no pedagogos. Magis, Revista Internacional de Investigación en Educación, 13, 1-22. https://doi: 10.11144/ Javeriana.m13.mecp

Sotomayor, C., \& Gysling, J. (2011). Estándares y regulación de calidad de la formación de profesores: discusión del caso chileno desde una perspectiva comparada. Calidad en la Educación, 35, 91-129. https://doi.org/10.31619/caledu.n35.97

Trujillo, J. (2014). El enfoque en competencias y la mejora de la educación. Ra Ximhai, 10(5), 307-322.

Valles, S. (2009). Técnicas cualitativas de investigación social. Reflexión metodológica y práctica profesional. Madrid: Editorial Síntesis.

Vasilachis de Gialdino, I. (2006). (Coord.) Estrategias de investigación cualitativa. España: Editorial Barcelona. 